\title{
Energetics and electronic structure of nanoscale rotors consisting of triptycene and hydrocarbon molecules
}

\author{
Miki Akiba and Susumu Okada*
}

Graduate School of Pure and Applied Sciences, University of Tsukuba, 1-1-1 Tennodai, Tsukuba, Ibaraki 305-8571, Japan

\begin{abstract}
Using the density functional theory with generalized gradient approximation, we studied the energetics and electronic structures of nanoscale rotors consisting of tryptycene and hydrocarbon molecules with respect to their mutual orientation. Energy barriers for the rotational motion of an attached hydrocarbon molecule range from 40 to $200 \mathrm{meV}$, depending on the attached molecular species and arrangements. The electronic structure of the nanoscale molecular rotors does not depend on the rotational angle of the attached hydrocarbon molecules.
\end{abstract}

\section{Introduction}

Triptycene is an aromatic hydrocarbon molecule with $D_{3 h}$ symmetry and a Y-shaped ridged structure consisting of three benzene rings connected via bridgehead $\mathrm{C}$ atoms with fourfold coordination ( $\mathrm{sp}^{3}$ hybridization) situated at the molecular axis. ${ }^{1)}$ In addition to triptycene, the molecules possessing acene panels with $\mathrm{sp}^{3} \mathrm{C}$ bridgeheads have been synthesized experimentally and known as iptycene, which is characterized by the size and shape of three acene planes. ${ }^{2-4)}$ The ridged three-dimensional structures of iptycene, especially triptycene and pentiptycene, allow them constituent units of covalent organic frameworks for atoms and molecular storages, ${ }^{5-9)}$ supermolecules for a hybrid structure with other molecules, ${ }^{10,11)}$ and molecular machines. ${ }^{12-14)}$ The oligomerization and polymerization of triptycene result in porous and covalent architectures, exhibiting an unusual electronic structure. Triptycene and its derivatives self-assembly form condensed phases in which triptycene and its derivatives act as hosts for foreign molecules and atoms forming host-guest complexes with interesting structural hierarchy, being expected to result in unusual physical properties that are absent in the simple superposition of that of constituents as in the case of complexes consisting of nanocarbon materials. ${ }^{15-18)}$

${ }^{*}$ E-mail: sokada@comas.frsc.tsukuba.ac.jp 
Triptycene is a key material for designing molecular machines owing to its highsymmetry molecular shape. By attaching other triptycene or hydrocarbon molecules to the bridgehead $\mathrm{C}$ atoms via a $\mathrm{C}$ chain, we can construct molecular rotors with nanometer scale as a key component of molecular machines. For instance, the threefold symmetrical shape of the molecule makes the triptycene complexes molecular gears for conveying mechanical motion in nanoscale machines, by rotating around $\mathrm{C}$ chains connecting constituents as in the case of the polyarenes. Despite several triptycene derivatives have been synthesized as the potential candidate for the nanoscale rotors, the microscopic mechanism of the rotational motion, the energetics, and the electronic structure of such derivatives are still unclear. Thus, in this paper, we aim to investigate the energetics and electronic structures of molecular complexes consisting of triptycene and hydrocarbon molecules connected via a linear $\mathrm{C}$ chain, by conducting first-principles total-energy calculations within the framework of the density functional theory (DFT). Our calculations show that the attached hydrocarbon molecules prefer the molecular orientation in which their acene panels are separated from the acene panels of triptycene as much as possible. The potential energy barrier for the rotational motion of attached molecules strongly depends on the molecular species and mutual arrangement. In contrast, the electronic structure of nanoscale rotors is insensitive to the mutual molecular arrangement of the attached hydrocarbon molecules.

\section{Calculation method and structural model}

All calculations were performed using DFT ${ }^{19,20)}$ implemented in the STATE package. ${ }^{21)}$ For the calculation of the exchange-correlation energy among interacting electrons, we used the generalized gradient approximation (GGA) including the spin degree of freedom with functional forms of Perdew-Burke-Ernzerhof. ${ }^{22,23)}$ Ultrasoft pseudopotentials generated with the Vanderbilt scheme were used to describe electron-ion interactions. ${ }^{24)}$ The valence wave functions and deficit charge density were expanded using plane-wave basis sets with cutoff energies of 25 and $225 \mathrm{Ry}$, respectively, which give sufficient convergence in both geometric and electronic structures of carbon derivatives. ${ }^{25,26)}$ Structural optimizations were performed for each rotational angle until the remaining forces on each atom were less than $5 \mathrm{mRy} / \AA$. To simulate isolated molecular complexes consisting of triptycene and hydrocarbon molecules, the complexes are situated in a large cubic cell of which cell parameter is $17 \AA$. Brillouin-zone integration was performed with the $\Gamma$ point sampling for self-consistent geometric and electronic structure calculations. 

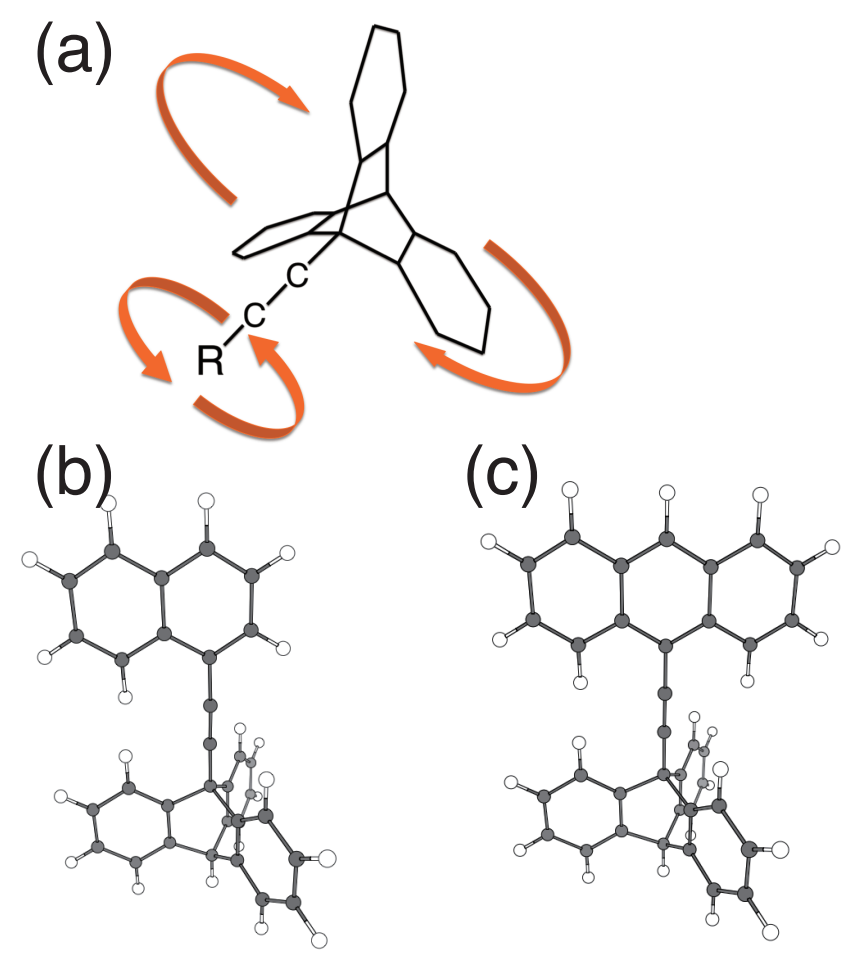

(d)

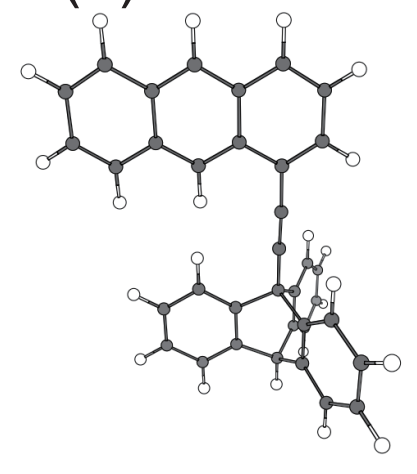

(e)

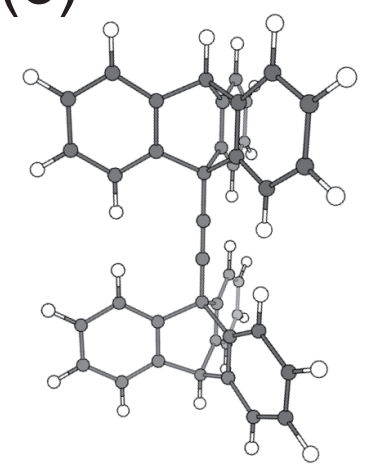

Fig. 1. (a) A schematic structural model of a nanoscale rotor consisting of triptycene and hydrocarbon molecules $R$, where $R$ represent (b) naphthalene, (c) anthracene with the symmetric arrangement, (d) anthracene with the asymmetric arrangement, and (e) triptycene. The gray and white circles denote $\mathrm{C}$ and $\mathrm{H}$ atoms, respectively.

In this work, we consider nanoscale rotors consisting of triptycene and the hydrocarbon molecules, e.g. naphthalene, anthracene, and triptycene, which are connected via $\mathrm{C}_{2}$ alkyne to avoid the steric hindrance of the rotational motions (Fig. 1). The $\mathrm{C}_{2}$ alkyne unit is attached to a $\mathrm{sp}^{3} \mathrm{C}$ bridgehead of triptycene by replacing the $\mathrm{H}$ atom. These hydrocarbon molecules are attached to triptycene, in which the acene panels are arranged in parallel conformations to that of triptycene. For anthracene, we consider 


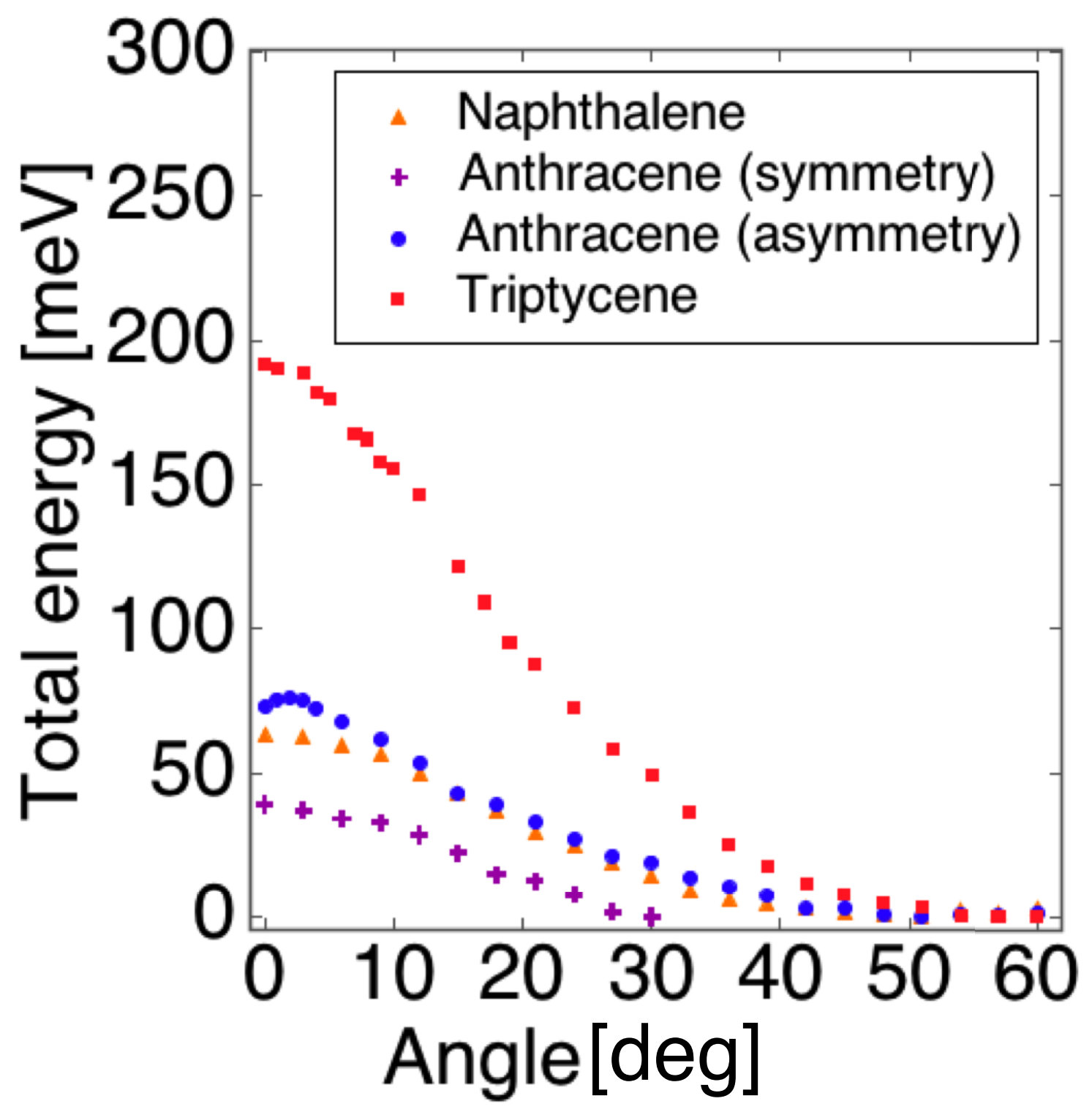

Fig. 2. Relative total energies of the nanoscale rotors consisting of triptycene and the hydrocarbon molecules $R$, where $R$ represent naphthalene, anthracene with the symmetric arrangement, anthracene with the asymmetric arrangement, and triptycene with respect to the energy of these complexes in their most stable conformation. The triangles, crosses, circles, and squares denote the energies of the molecular rotors containing naphthalene, anthracene with the symmetric arrangement, anthracene with the asymmetric arrangement, and triptycene, respectively.

the symmetric and asymmetric arrangements with respect to the three $\mathrm{C}_{6}$ rings. Owing to the molecular conformation of the complexes, the attached molecules can rotate along the bridging $\mathrm{C}_{2}$ unit as the nanoscale rotors. 


\section{Results and discussion}

Figure 2 shows the relative total energy of the nanoscale rotors as a function of the mutual rotational angle with respect to the total energy of their stable conformation. Under the stable conformation, the attached molecules prefer the rotational angle in which their acene panels are separated from the acene panels of triptycene as much as possible. In contrast, the attached molecules do not prefer the arrangement in which their acene panels are situated above the acene panels of triptycene. The relative stability in terms of the rotation angles are ascribed to the Coulomb repulsive interaction between positively charged $\mathrm{H}$ atoms attached to the acene panels. Thus, the total energy monotonically decreases with increasing rotational angle for all complexes.

To induce the rotational motion of the attached molecules, it is necessary to overcome the energy barriers arising from the repulsive interaction between $\mathrm{H}$ atoms. The calculated energy barriers for the rotational motion are 63.09, 76.10, 39.09, and 192.13 $\mathrm{meV}$ for naphthalene, anthracene with the asymmetric arrangement, anthracene with the symmetric arrangement, and triptycene, respectively. This fact indicates that the rotational motion of the nanorotors strongly depends on the constituent hydrocarbon molecules. It is notable that the barrier for triptycene is approximately three times higher than that for naphthalene, corroborating that the Coulomb repulsive interaction is the physical origin of the energy barrier because triptycene possesses three acene panels. Among the attached molecules, anthracene with the symmetric arrangement has the lowest energy barrier for the rotation, while triptycene has the largest barrier.

Figure 3 shows the electronic energy level near the Fermi level and the isosurfaces of the squared wave function of the highest occupied (HO) and the lowest unoccupied (LU) states of the nanorotors with the mutual angles of 0 degree. Because a $\pi$ electron system of triptycene consists of three isolated $\mathrm{C}_{6}$ rings connected to bridgehead $\mathrm{sp}^{3}$ $\mathrm{C}$ atoms, triptycene has a large HO-LU gap, although it has $18 \pi$ electrons on the covalent network. Thus, the nanoscale rotors have a narrower HO-LU gap than that of triptycene, owing to the extended nature of the $\pi$ electrons of naphthalene and anthracene. As shown in Fig. 3, the wave function of HO and LU states is distributed on the attached naphthalene and anthracene portions. It is noteworthy that the HO-LU gap of nanoscale rotors is slightly narrower than that of an isolated naphthalene and anthracene, owing to spilled $\pi$ electrons on the interconnect alkyne.

In contrast to the energetics with respect to the rotational motion of the attached 
(a)

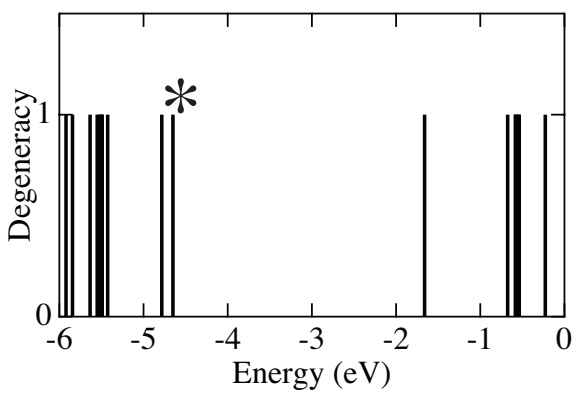

(c)

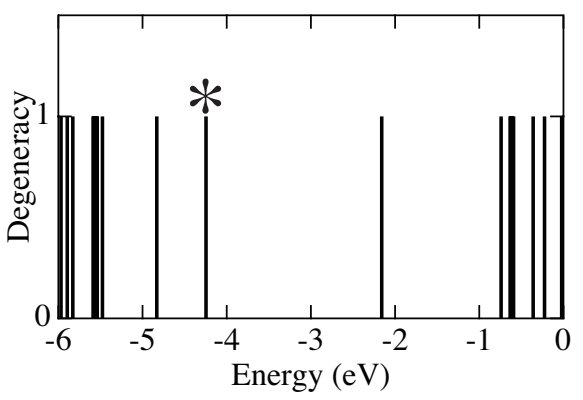

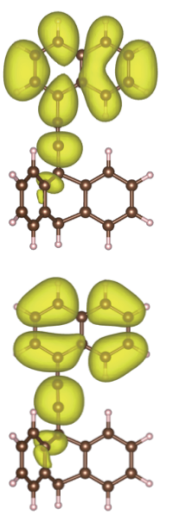

(b)
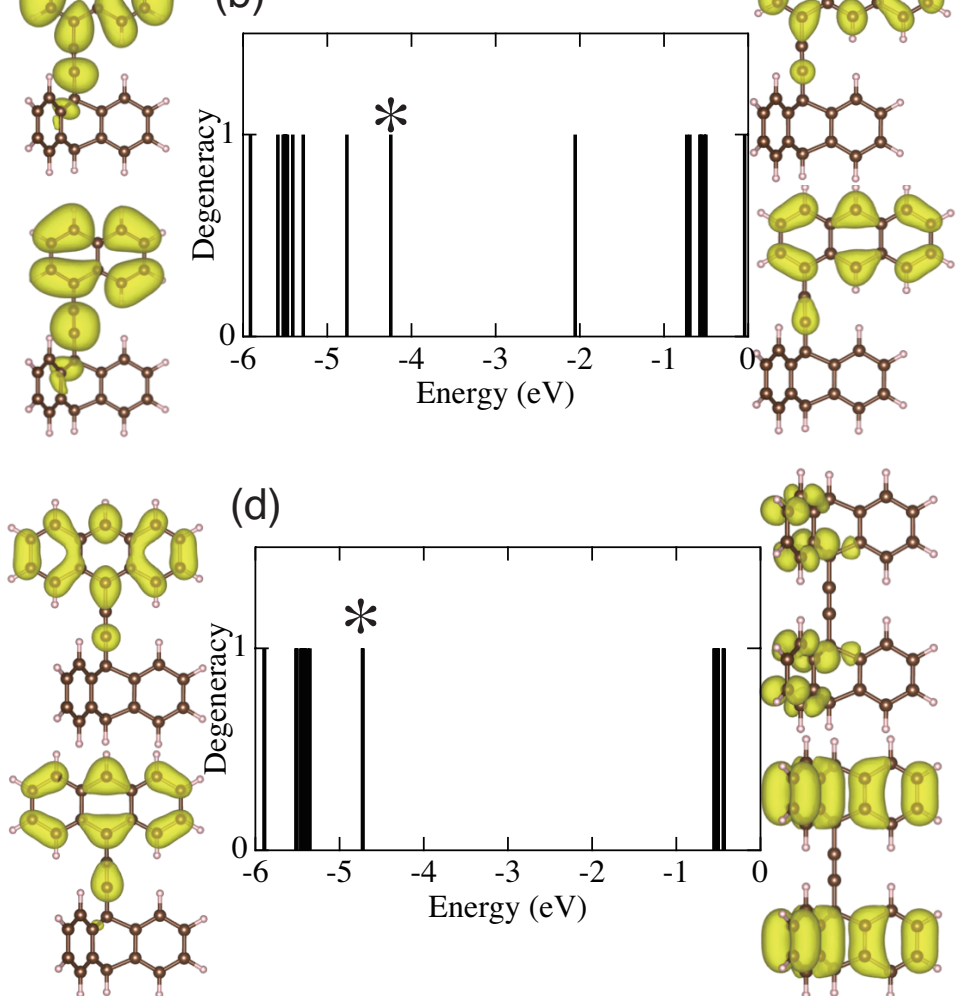

(d)

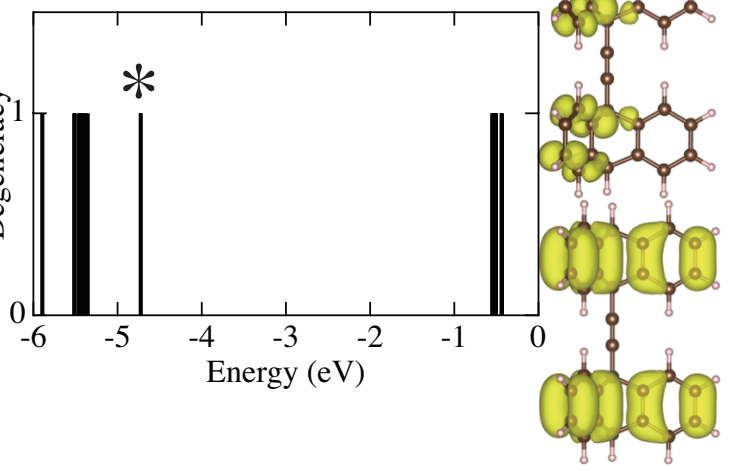

Fig. 3. Electronic energy levels near the Fermi level and isosurfaces of squared wave functions of the $\mathrm{HO}$ and LU states of the nanoscale rotors consisting of triptycene and the hydrocarbons $R$, where $R$ represent (a) naphthalene, (b) anthracene with the symmetric arrangement, (c) anthracene with the asymmetric arrangement, and (d) triptycene. The energies are measured from that of the vacuum level. The asterisk denotes the HO state.

molecules, the electronic structure of the nanoscale rotors is insensitive to the rotational motion (Fig. 4). For all molecular rotors, the electronic energy levels near the HO-LU gap retain their energies against the rotation. This fact indicates that the $\pi$ electron systems on each constituent, triptycene and the attached hydrocarbon molecules, are perfectly separated from each other by bridgehead $\mathrm{sp}^{3} \mathrm{C}$ atoms situated at the molecular axis of triptycene. This fact implies that the triptycene derivative is not suitable for the mechanoelectronic applications. On the other hand, the structurally robust electronic structure of the hybrids allows them a constituent for the sensing devices. However, note that the LU and $\mathrm{HO}$ states of the attached hydrocarbon molecules show the downward shift by attaching to triptycene, because of the extent of the $\pi$ electron to the interconnect $\mathrm{C}_{2}$ unit between them. 

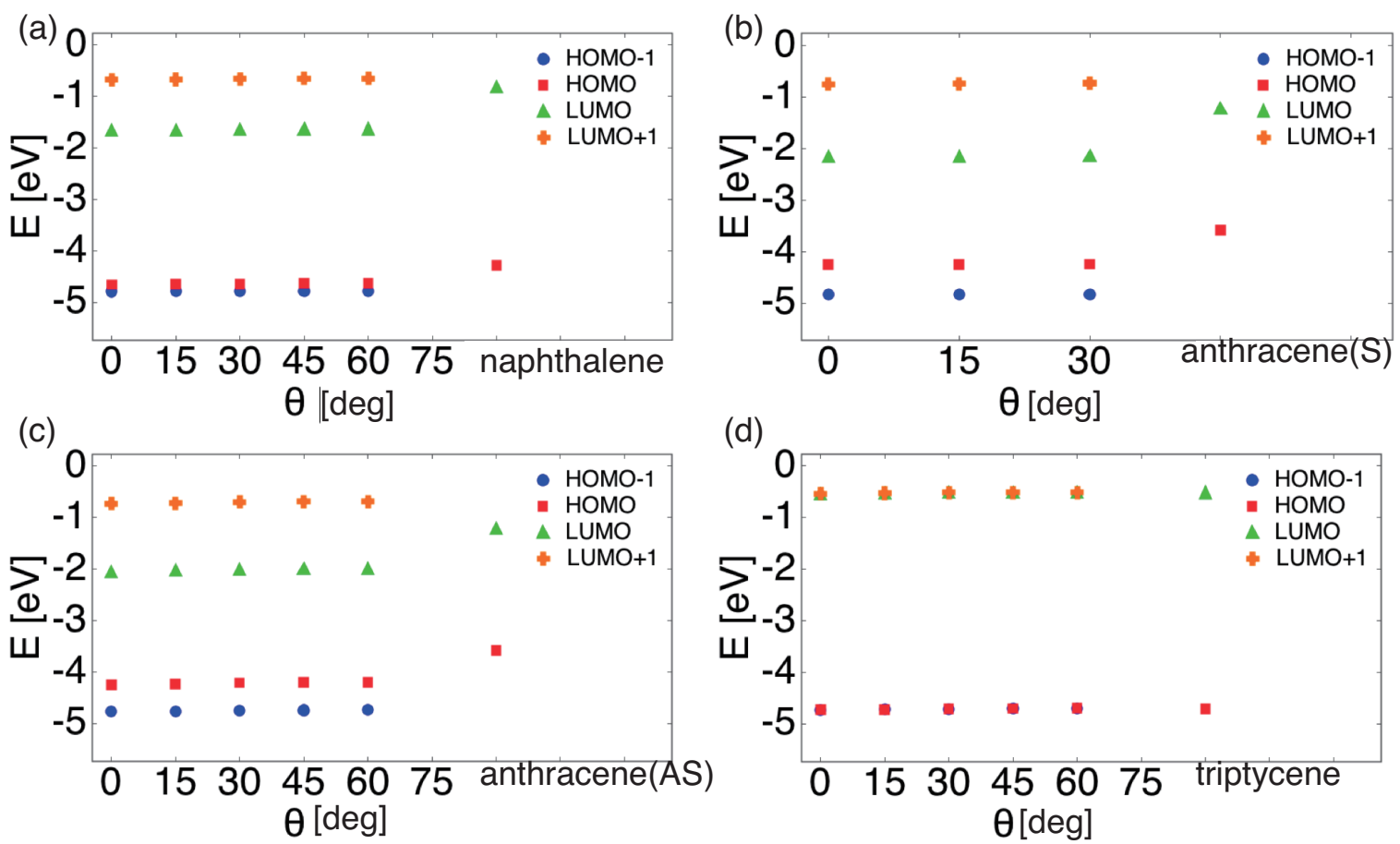

Fig. 4. Electronic energy levels near the Fermi level of the nanoscale rotors consisting of triptycene and hydrocarbon molecules $R$, where $R$ represent (a) naphthalene, (b) anthracene with the symmetric arrangement, (c) anthracene with the asymmetric arrangement, and (d) triptycene as functions of the rotational angle $\theta$. The energies are measured from that of the vacuum level. The crosses, triangles, squares, and circles denote $\mathrm{LU}+1, \mathrm{LU}, \mathrm{HO}$, and $\mathrm{HO}-1$ states, respectively.

\section{Summary}

Using DFT with GGA, we studied the energetics and electronic structure of nanoscale rotors consisting of tryptycene and the hydrocarbon molecules naphthalene, anthracene, and triptycene, with respect to their mutual orientation. The energy barrier for the rotational motion of the attached hydrocarbon molecules is sensitive to the molecular species attached to triptycene via the $\mathrm{C}_{2}$ chain. The calculated energy barriers for the rotational motion are 63.09, 76.10, 39.09, and $192.13 \mathrm{meV}$ for naphthalene, anthracene with the asymmetric arrangement, anthracene with the symmetric arrangement, and triptycene, respectively, indicating that the attached molecules oscillate around the energy minima under room temperature. The energy barrier is ascribed to the Coulomb repulsive interaction between $\mathrm{H}$ atoms attached to triptycene and hydrocarbon molecules. In contrast, the electronic structure of the nanoscale rotors is insensitive to the mutual molecular arrangement of the attached hydrocarbon molecules. The formation of the complexes leads to the downward shift of the electron states associated with the 
attached hydrocarbon owing to the extent of the $\pi$ states on the $\mathrm{C}_{2}$ linker.

\section{Acknowledgments}

This work was supported by JST-CREST Grant Number JPMJCR1532 from the Japan Science and Technology Agency, JSPS KAKENHI Grant Numbers JP17H01069, JP16H00898, and JP16H06331 from the Japan Society for the Promotion of Science, and the Joint Research Program on Emission Energy Research, Institute of Advanced Energy, Kyoto University. A part of the calculations was performed on an NEC SX-Ace at the Cybermedia Center at Osaka University and on an SGI ICE XA/UV at the Institute of Solid State Physics, The University of Tokyo. 


\section{References}

1) P. D. Barlett, M. J. Ryan, and S. G. Cohen, J. Am. Chem. Soc. 64, 2649 (1942).

2) H. Hart, S. Shamouilian, and Y. Takehira, J. Org. Chem. 46, 4427 (1981).

3) T. M. Swager, Acc. Chem. Res. 41, 1181 (2008).

4) J. H. Chong and M. J. MacLachlan, Chem. Soc. Rev. 38, 3301, (2009).

5) C. Zhang and C. F. Chen, Crystengcommunication 12, 3266 (2010).

6) C. Zhang and C. F. Chen, J. Org. Chem. 72, 3880 (2007).

7) C. Zhang and C. F. Chen, J. Org. Chem. 72, 9339 (2007).

8) M. Xue and C. F. Chen, Chem. Comm. 47, 2318 (2011).

9) J. Sorimachi and S. Okada, Phys. Rev. B 96, 024103 (2017).

10) E. M. Veen, P. M. Postma, H. T. Jonkman, A. L. Spek, and B. L. Feringa, Chem. Commum., 17, 1709 (1999).

11) X. H. Tian and C. F. Chen, Chem. Eur. J. 16, 8072 (2010).

12) G. Jimenez-Bueno and G. Rapenne, Tetrahedron Lett. 446261 (2003).

13) L. Grill, K. H. Rieder, F. Moresco, G. Rapenne, S. Stojkovic, X. Bouju, and C. Joachim, Nat. Nanotechnol. 295 (2007).

14) G. Rapenne and G. Jimenez-Bueno, Tetrahedron 637018 (2007).

15) J. -C. Charlier, X. Gonze, and J. -P. Michenaud, Europhys. Lett. 29, 43 (1995).

16) P. Delaney, H. J. Choi, J. Ihm, S. G. Louie, and M. L. Cohen, Nature 391, 466 (1998).

17) S. Saito and A. Oshiyama, Phys. Rev. Lett. 66, 2637 (1991).

18) S. Okada, S. Saito, and A. Oshiyama, Phys. Rev. Lett. 86, 3835 (2001).

19) P. Hohenberg and W. Kohn, Phys. Rev. 136, B864 (1964).

20) W. Kohn and L. J. Sham, Phys. Rev. 140, A1144 (1965).

21) Y. Morikawa, K. Iwata, and K. Terakura, Appl. Surf. Sci. 169-170, 11 (2001).

22) J. P. Perdew, K. Burke, and M. Ernzerhof, Phys. Rev. Lett. 77, 3865 (1997).

23) J. P. Perdew, K. Burke, and M. Ernzerhof, Phys. Rev. Lett. 78, 1396 (1997).

24) D. Vanderbilt, Phys. Rev. B 41, 7892 (1990).

25) M. Maruyama, N.T. Cuong, and S. Okada, Carbon 109, 755 (2016).

26) M. Maruyama, N.T. Cuong, and S. Okada, J. Phys. Soc. Jpn. 84, 084706 (2015). 